\title{
Pi-coefficient analysis of update algorithms for adaptive systems*
}

\author{
Jia-Sheng Heh and I-Kong Fong \\ Department of Electrical Engineering, National Taiwan University, Taipei, Taiwan 10617, ROC
}

Jinn-Fwu Wu

Chung-Shan Institute of Science and Technology, Tao-Yuan, Taiwan 32500, ROC

Received 14 February 1992

Revised 16 July 1992

\begin{abstract}
This paper finds the appropriate pi-coefficients for a parameter estimation adaptive system and uses them to analyze the stability of two estimation algorithms. The estimation error dynamics of the system are modeled by a linear time-invariant subsystem and a nonlinear time-varying update law in a feedback loop. Then the so-called max- $p$ problems are formulated and solved to obtain the pi-coefficients for the linear subsystem and nonlinear update law. For the investigated system, the quantitative results show that the least-squares update algorithm has larger stability range than that of the gradient algorithm, and the $\sigma$-modification scheme gives larger stability ranges for both algorithms.
\end{abstract}

Keywords: Pi-sharing theory; finite mean-square gain; strictly positive realness; stability; adaptive system.

\section{Introduction}

The pi-sharing theory [5] uses a set of pi-coefficients to describe the generalized concept of energy distributions of a system, such as passivity or dissipativeness. With different constraints, these coefficients represent various stability conditions quantitatively. The composite pi-coefficients of a feedback system can be determined by the pi-coefficients of its subsystems. By choosing proper coefficients, it is possible to get less conservative conclusions about the system stability than using the conventional stability conditions, such as the strictly positive realness (SPR) condition.

Towards the objective of maximizing the finite mean-square gain (FMSG) stability range, the max-p problem $[3,6,7]$ is formulated for finding the optimal pi-coefficients. In [7], the optimal constant picoefficients of the second-order linear systems are derived, and applied to the sampling time determination problem of an adaptive control system.

This paper applies the pi-sharing theory to the stability analysis problem of a parameter estimation adaptive system [1]. The estimation error dynamics of the system are modeled by a linear time-invariant (LTI) subsystem and a nonlinear time-varying (NTV) update law in a feedback loop. Then the max-p problems [6] are formulated and solved to obtain the pi-coefficients for both the LTI subsystem and the NTV update law. From the investigated system, the quantitative results show that the least-squares update algorithm has larger stability range than that of the gradient algorithm, and the $\sigma$-modification scheme gives larger stability ranges for both algorithms.

\footnotetext{
Correspondence to: Dr. Jinn-Fwu Wu, Chung-Shan Institute of Science and Technology, Tao-Yuan, Taiwan 32500, ROC

* This research was supported by the National Science Council of the Republic of China under Grant NSC 81-0404-E002-007.
} 


\section{The optimal pi-coefficients}

A single-input-single-output 'factorable' discrete-time system [5]

$$
\begin{aligned}
& \boldsymbol{x}(k+1)=\boldsymbol{A}(k) \boldsymbol{x}(k)+\boldsymbol{b}(k) u(k), \\
& y(k)=\boldsymbol{c}^{\Upsilon}(k) \boldsymbol{x}(k)+d(k) u(k),
\end{aligned}
$$

where $k \in \mathbb{N}, \boldsymbol{x}(k) \in \mathbb{R}^{n}, u(k) \in \mathbb{R}, y(k) \in \mathbb{R}$, and $\boldsymbol{A}, \boldsymbol{b}, \boldsymbol{c}, d$ are system 'matrices' with appropriate dimensions, is said to be pi-sharing with respect to pi-coefficients $\{\boldsymbol{\Gamma}(k), \boldsymbol{Q}(k), p(k), r(k)\}$ if for all $\tau \in \mathbb{N}$,

$$
\begin{aligned}
\sum_{k=0}^{\tau} u(k) y(k) \geq & \boldsymbol{x}^{\mathrm{T}}(\tau+1) \Gamma(\tau+1) \boldsymbol{x}(\tau+1)-\boldsymbol{x}^{\mathrm{T}}(0) \Gamma(0) \boldsymbol{x}(0)+\sum_{k=0}^{\tau} \boldsymbol{x}^{\mathrm{T}}(k) \boldsymbol{Q}(k) \boldsymbol{x}(k) \\
& +\sum_{k=0}^{\tau} y^{2}(k) p(k)+\sum_{k=0}^{\tau} u^{2}(k) r(k),
\end{aligned}
$$

where $\boldsymbol{\Gamma}(k)$ and $\boldsymbol{Q}(k)$ are positive-semidefinite (p.s.d., denoted as $\geq \mathbf{0}$ hereafter) matrices for $k \leq \tau+1$. Different ranges of the pi-coefficients correspond to various stabilities, one of which is the FMSG stability. System (1) is said to preserve FMSG stability if there exist two finite real numbers $\varepsilon_{1}$ and $\varepsilon_{2}$ such that $\sum_{k=0}^{\tau} y^{2}(k) \leq \varepsilon_{1} \sum_{k=0}^{\tau} u^{2}(k)+\varepsilon_{2} \boldsymbol{x}^{\mathrm{T}}(0) \boldsymbol{x}(0)$, for all $u(\cdot): \mathbb{N} \rightarrow \mathbb{R}, \boldsymbol{x}(0) \in \mathbb{R}^{n}$, and $\tau \in \mathbb{N}$. The equivalent constraints on pi-coefficients have been proven [5] to be that there exist $r_{0} \in \mathbb{R}$ and $p_{0}>0$ such that

$$
p(k) \geq p_{0}, r(k) \geq r_{0}, \quad \forall k .
$$

However, the choice of pi-coefficients is not unique, and inappropriate selections may lead to conservative, or even useless, conclusions. In [5] there is a sufficient condition for 'legal' pi-coefficients:

$$
\boldsymbol{M}(k)=\left[\begin{array}{cc}
\boldsymbol{M}_{1}(k) & \boldsymbol{m}_{2}(k) \\
\boldsymbol{m}_{2}^{\mathrm{T}}(k) & m_{4}(k)
\end{array}\right]
$$

must be negative-semidefinite (n.s.d., denoted as $\leq \mathbf{0}$ hereafter) for all $k$, where

$$
\begin{aligned}
& \boldsymbol{M}_{1}(k)=\boldsymbol{A}^{\mathrm{T}}(k) \Gamma(k+1) \boldsymbol{A}(k)-\boldsymbol{\Gamma}(k)+\boldsymbol{Q}(k)+p(k) \boldsymbol{c}(k) \boldsymbol{c}^{\mathrm{T}}(k), \\
& \boldsymbol{m}_{2}(k)=\boldsymbol{A}^{\mathrm{T}}(k) \Gamma(k+1) \boldsymbol{b}(k)-\frac{1}{2} \boldsymbol{c}(k)+p(k) d(k) \boldsymbol{c}(k), \\
& m_{4}(k)=\boldsymbol{b}^{\mathrm{T}}(k) \Gamma(k+1) \boldsymbol{b}(k)-d(k)+r(k)+p(k) d^{2}(k) .
\end{aligned}
$$

For the composite system $\Xi_{c}$ in Figure 1, suppose the subsystem $\Xi_{i}$ has a set of pi-coefficients $\left\{\boldsymbol{\Gamma}_{i}(\cdot), \boldsymbol{Q}_{i}(\cdot)\right.$, $\left.p_{i}(\cdot), r_{i}(\cdot)\right\}, i=1,2$. Then it is easy to see that $\Xi_{c}$ has FMSG stability if $r_{1}(k)$ and $p_{2}(k)$ are finite for all $k \in \mathbb{N}$, and

$$
\begin{aligned}
& \inf _{k \in \mathbb{N}} r_{1}(k)+p_{2}(k)>0, \\
& \inf _{k \in \mathbb{N}} p_{1}(k)+r_{2}(k)>0 .
\end{aligned}
$$

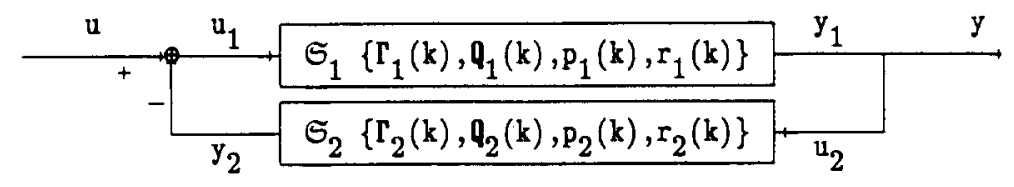

Fig. 1. A feedback system $\Xi_{\mathrm{c}}$ with subsystems $\Xi_{1}$ and $\Theta_{2}$. 
Thus, to maximize the FMSG stability range for the system $\Xi_{c}$, we would like to find the pi-coefficients for $\Xi_{i}$ 's with $p_{i}$ 's and $r_{i}$ 's as large as possible, under the conditions $\Gamma_{i}(k) \geq \mathbf{0}, \boldsymbol{Q}_{i}(k) \geq \mathbf{0}$, and $\boldsymbol{M}(k)$ 's $\leq \mathbf{0}$, for $i=1,2$. Now, from (2) it is clear that $p$ 's and $r$ 's cannot be large simultaneously. Hence, we may choose to maximize the coefficient $p$, requiring $r(k)$ to be greater than or equal to a parameter $r_{0}$. This forms the max-p problem:

$$
\begin{array}{ll}
\underset{\Gamma(k), \boldsymbol{Q}(k), r(k)}{\operatorname{Maximize}} & p(k) \\
\text { subject to } & \boldsymbol{\Gamma}(k) \geq \mathbf{0}, \quad \boldsymbol{Q}(k) \geq \mathbf{0}, \quad r(k) \geq r_{0}, \\
& \boldsymbol{M}(k) \leq \mathbf{0} .
\end{array}
$$

Although in [7] we have shown that the optimal solutions to (6) occur at $Q(k)=\mathbf{0}$ and $r(k)=r_{0}$, which largely simplifies the problem, the general solutions are still hard to find. For LTI systems with order $\leq 2$, i.e., with the transfer function

$$
H\left(z^{-1}\right)=\kappa \frac{1+b_{1} z^{-1}+b_{2} z^{-2}}{1-a_{1} z^{-1}-a_{2} z^{-2}},
$$

a set of time-invariant suboptimal solutions are given in [7] for $\boldsymbol{\Gamma}$ restricted to be diagonal. The solutions are derived by applying the method developed in [6].

\section{The parameter estimation adaptive system}

Consider the parameter estimation adaptive system shown in Figure 2, where

$$
y(k+1)=\boldsymbol{\theta}^{\mathrm{T}} \boldsymbol{\psi}(k)
$$

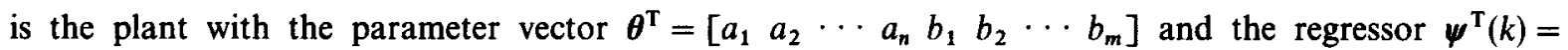
$[y(k) \cdots y(k-n+1) u(k) \cdots u(k-m+1)]$. The estimated output is

$$
\hat{y}(k+1)=\hat{\theta}^{\mathrm{T}}(k+1) \phi(k),
$$

with the estimated parameters and regressor, respectively, being

$$
\hat{\boldsymbol{\theta}}^{\mathrm{T}}(k+1)=\left[\hat{a}_{1}(k+1) \cdots \hat{a}(k+1) \hat{b}_{1}(k+1) \cdots \hat{b}_{m}(k+1)\right]
$$

and

$$
\phi^{\mathrm{T}}(k)=[\hat{y}(k) \cdots \hat{y}(k-n+1) u(k) \cdots u(k-m+1)] .
$$

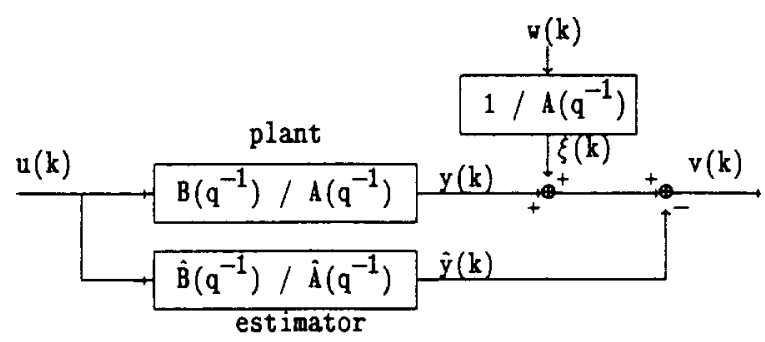

Fig. 2. A parameter estimation adaptive system. 


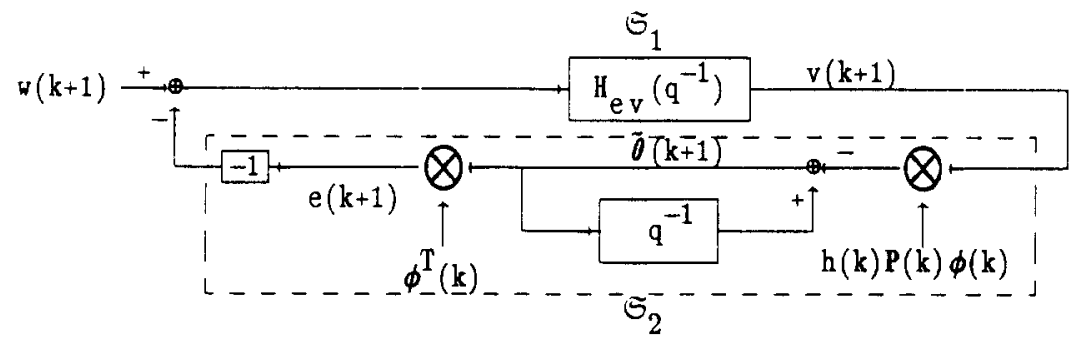

Fig. 3. The error model.

The update rule for the parameters chosen in [5] is the least-squares algorithm:

$$
\hat{\boldsymbol{\theta}}(k+1)+\hat{\boldsymbol{\theta}}(k)+h(k) \boldsymbol{P}(k) \boldsymbol{\phi}(k) v(k+1)
$$

where $h(k)>0$ is the step size, the covariance matrix $\boldsymbol{P}(k)$ iterates through

$$
\boldsymbol{P}^{-1}(k+1)=\gamma(k) \boldsymbol{P}^{-1}(k)+\delta(k) \phi(k) \phi^{\mathrm{T}}(k), \quad 0 \leq \gamma(k) \leq 1,0 \leq \delta(k)<\infty,
$$

and $v(k+1)=y(k+1)-\hat{y}(k+1)+\xi(k+1)$ with output noise $\xi(k+1)$.

Let the parameter error be $\tilde{\boldsymbol{\theta}}(k+1)=\boldsymbol{\theta}-\hat{\boldsymbol{\theta}}(k+1)$. Then the estimation error is

$$
e(k+1)=\boldsymbol{\theta}^{\mathrm{T}} \phi(k)-\hat{\boldsymbol{\theta}}^{\mathrm{T}}(k+1) \phi(k)=\tilde{\boldsymbol{\theta}}^{\mathrm{T}}(k+1) \phi(k) .
$$

Denote $q^{-1}$ as the delay operator. The error model shown in Figure 3 can be obtained by introducing an 'input noise' $w(k+1)=A\left(q^{-1}\right) \xi(k+1)$, where $A\left(q^{-1}\right)$ is defined below. The LTI feedforward subsystem $\Xi_{1}$ comes from the relation between $e(\cdot), v(\cdot)$ and $w(\cdot)[5]$ :

$$
v(k+1)=H_{e v}\left(q^{-1}\right)[e(k+1)+w(k+1)]
$$

where

$$
H_{e v}\left(z^{-1}\right)=\frac{1}{A\left(z^{-1}\right)}=\frac{1}{1-a_{1} z^{-1}-a_{2} z^{-2} \cdots-a_{n} z^{-n}} .
$$

The parameter error and the update rule constitute the NTV feedback subsystem $\Xi_{2}$, which can be described by the 'state equations' [5]

$$
\tilde{\boldsymbol{\theta}}(k+1)=\boldsymbol{A}_{2}(k) \tilde{\boldsymbol{\theta}}(k)+\boldsymbol{b}_{2}(k) v(k+1), \quad-e(k+1)=c_{2}^{\mathrm{T}}(k) \tilde{\boldsymbol{\theta}}(k)+d_{2}(k) v(k+1),
$$

where

$$
\left\{\boldsymbol{A}_{2}(k), \boldsymbol{b}_{2}(k), c_{2}^{\mathrm{T}}(k), d_{2}(k)\right\}=\left\{\boldsymbol{I},-h(k) \boldsymbol{P}(k) \phi(k),-\boldsymbol{\phi}^{\mathrm{T}}(k), h(k) \boldsymbol{\phi}^{\mathrm{T}}(k) \boldsymbol{P}(k) \phi(k)\right\} .
$$

To analyze the stability of this feedback system by the pi-sharing theory, Lawrence and Johnson [5] choose the following pi-coefficients:

$$
\begin{aligned}
& \left\{\boldsymbol{\Gamma}_{1}, \boldsymbol{Q}_{1}, p_{1}, r_{1}\right\}=\left\{\operatorname{diag}[n \alpha,(n-1) \alpha, \ldots, \alpha], \frac{1}{2} \alpha \boldsymbol{I}, \frac{1}{2}-n \alpha, \frac{1}{2}\right\}, \\
& \left\{\boldsymbol{\Gamma}_{2}(k), \boldsymbol{Q}_{2}(k), p_{2}(k), r_{2}(k)\right\}=\left\{\boldsymbol{P}^{-1}(k),[1-\gamma(k)] \boldsymbol{P}^{-1}(k),-\delta(k), \frac{\boldsymbol{\phi}^{\mathrm{T}}(k) \boldsymbol{P}(k) \boldsymbol{\phi}(k)}{4 \gamma(k)}\right\},
\end{aligned}
$$


where $\alpha=\sum_{i=1}^{n} a_{i}^{2}$ and $h(k)=1 /(2 \gamma(k))$. Then the FMSG stability conditions are:

$$
\begin{aligned}
& \inf _{k}\left\{r_{1}+p_{2}(k)\right\}=\frac{1}{2}-\sup _{k} \delta(k)>0, \\
& \inf _{k}\left\{p_{1}+r_{2}(k)\right\}=\frac{1}{2}-n \alpha+\inf _{k} \frac{\phi^{\mathrm{T}}(k) P(k) \phi(k)}{4 \gamma(k)}>0,
\end{aligned}
$$

where $(16 b)$ is a signal condition of the regressor. If

$$
p_{1}=\frac{1}{2}-n \alpha<0,
$$

the regressor vector $\phi(k)$ has to satisfy

$$
\inf _{k} \frac{\phi^{\mathrm{T}}(k) \boldsymbol{P}(k) \phi(k)}{4 \gamma(k)}>\left|\frac{1}{2}-n \alpha\right|
$$

to ensure the FMSG stability.

Take $n=2$ as an example. Then

$$
H_{e v}\left(z^{-1}\right)=\frac{1}{1-a_{1} z^{-1}-a_{2} z^{-2}}
$$

whose SPR condition [4] is

$$
1+a_{1}-a_{2}>0, \quad 1-a_{1}-a_{2}>0, \text { and } 1+3 a_{2}>0 \text { or } a_{1}^{2}+8 a_{2}+8 a_{2}^{2}<0 .
$$

With $a_{1}=-0.85$ and $a_{2}=-0.10$, it is clear that $H_{e v}\left(z^{-1}\right)$ is SPR. By the passivity theorem [1], this system is FMSG-stable, no matter what $\phi(k)$ is. However, from $(17), \phi(k)$ has to satisfy

$$
\inf _{k} \frac{\phi^{\mathrm{T}}(k) \boldsymbol{P}(k) \phi(k)}{4 \gamma(k)}>\left|\frac{1}{2}-n \alpha\right|=\left|\frac{1}{2}-2 \cdot\left(0.85^{2}+0.1^{2}\right)\right|=0.965
$$

to ensure the FMSG stability. So, (17) is more conservative than the passivity theorem. But, when we use the suboptimal pi-coefficients in $[7]$ for $\mathcal{S}_{1}$, i.e.,

$$
\left\{\boldsymbol{\Gamma}_{1}, \boldsymbol{Q}_{1}, p_{1}, \boldsymbol{r}_{1}\right\}=\left\{\left[\begin{array}{cc}
0.475-0.0475 r_{0} & 0 \\
0 & 0.05-0.005 r_{0}
\end{array}\right], \quad 0,0.05-0.0025 r_{0}, r_{0}\right\},
$$

the FMSG stability conditions become

$$
r_{0}-\sup _{k} \delta(k)>0 \text { and } 0.05-0.0225 r_{0}+\inf _{k} \frac{\phi^{\mathrm{T}}(k) P(k) \phi(k)}{4 \gamma(k)}>0 .
$$

An appropriate choice of $r_{0}$ can make all regressor vectors $\phi(k)$ satisfy (21).

Moreover, if $a_{1}=0.85$ and $a_{2}=-0.90$, it is known from (19) that $H_{e v}\left(z^{-1}\right)$ is not SPR. Then the passivity theory and the Lyapunov method fail. However, the suboptimal pi-coefficients [7] for $\Xi_{1}$,

$$
\left\{\Gamma_{1}, Q_{1}, p_{1}, r_{1}\right\}=\left\{\left[\begin{array}{cc}
0.875-1.3125 \cdot r_{0} & 0 \\
0 & 0.450-0.675 \cdot r_{0}
\end{array}\right], 0,-0.75-0.5625 r_{0}, r_{0}\right\},
$$

lead to the FMSG stability conditions

$$
r_{0}-\sup _{k} \delta(k)>0 \text { and } \inf _{k} \frac{\phi^{\mathrm{T}}(k) \boldsymbol{P}(k) \phi(k)}{4 \gamma(k)}>\left|0.75+0.5625 r_{0}\right| .
$$

From the above results, we see that, by choosing the appropriate pi-coefficients for a subsystem in a feedback configuration, we may obtain a less conservative conclusion about the FMSG stability. 


\section{Pi-coefficient analysis of the update algorithms}

In Section 3, though the suboptimal pi-coefficients [7] are used for $\Xi_{1}$ to get the less conservative results (21) and (22), these FMSG stability conditions are still based on the original pi-coefficients [5] for $\Xi_{2}$. To further improve the results we may try to find 'better' pi-coefficients for $\Xi_{2}$. However, the update rule (13) is an NTV system, which makes the corresponding max-p problem more difficult to solve. So, we add some more constraints to the max- $p$ problem for $\Theta_{2}$, and form the constrained max- $p$ problem:

$$
\begin{array}{ll}
\underset{r(k), \boldsymbol{Q}(k), r(k)}{\operatorname{Maximize}} & p(k) \\
\text { subject to } & \boldsymbol{\Gamma}(k) \geq \mathbf{0}, \quad \boldsymbol{Q}(k) \geq \mathbf{0}, \\
& \boldsymbol{M}_{\mathbf{1}}(k) \leq \mathbf{0}, \quad \boldsymbol{m}_{\mathbf{2}}(k)=0, \quad m_{\mathbf{4}}(k)=0 .
\end{array}
$$

As we shall see later, the value of $r(k)$ will be decided by the equality $m_{4}(k)=0$. Hence, there is no need to put an additional constraint for $r(k)$. The purpose of the constraints in $(23 \mathrm{c})$ is to form a more tractable mathematical problem. As it turns out, the solutions of this problem can give still more analytic results.

\subsection{The least-squares update law}

When the parameter update rule is the least-squares law (10), we have the following theorem.

Theorem 1. A feasible solution to (23), which may not be optimal, is

$$
\begin{aligned}
& \left\{\boldsymbol{\Gamma}_{2}(k+1), Q_{2}(k), p_{2}(k), r_{2}(k)\right\} \\
& \quad=\left\{\frac{1}{2} h^{-1}(k) \boldsymbol{P}^{-1}(k)-\eta^{*}(k) \phi(k) \phi^{\mathrm{T}}(k), \mathbf{0}, \eta^{*}(k), \frac{1}{2} h(k) \phi^{\mathrm{T}}(k) \boldsymbol{P}(k) \phi(k)\right\},
\end{aligned}
$$

where $\eta^{*}(k)$ is the largest $\eta(k)$ satisfying the following inequality:

$$
\frac{1}{2}\left[h^{-1}(k)-h^{-1}(k+1) \gamma(k)\right] \boldsymbol{P}^{-1}(k)-\left[\eta(k)+\frac{1}{2} h^{-1}(k+1) \delta(k)\right] \phi(k) \phi^{\mathrm{T}}(k) \geq 0 .
$$

Proof. From (4c) and (13), requiring $\boldsymbol{m}_{2}(k)=\mathbf{0}$ is equivalent to requiring

$$
\left[-\boldsymbol{\Gamma}_{2}(k+1) \cdot h(k) \cdot \boldsymbol{P}(k)+\frac{1}{2} \boldsymbol{I}-p_{2}(k) h(k) \boldsymbol{\phi}(k) \boldsymbol{\phi}^{\mathrm{T}}(k) \boldsymbol{P}(k)\right] \boldsymbol{\phi}(k)=\mathbf{0},
$$

which $\Gamma_{2}(k+1)=\frac{1}{2} h^{-1}(k) \boldsymbol{P}^{-1}(k)-p_{2}(k) \phi(k) \phi^{\mathrm{T}}(k)$ satisfies. From $(4 \mathrm{~d})$ and $\boldsymbol{P}(k)=\boldsymbol{P}(k)^{\mathrm{T}}$, requiring $m_{4}(k)=0$ is equivalent to requiring

$$
\begin{aligned}
& \frac{1}{2} h(k) \phi^{\mathrm{T}}(k) \boldsymbol{P}(k) \phi(k)-h(k) \phi^{\mathrm{T}}(k) \boldsymbol{P}(k) p_{2}(k) \phi(k) \phi^{\mathrm{T}}(k) h(k) \boldsymbol{P}(k) \phi(k) \\
& \quad-h(k) \phi^{\mathrm{T}}(k) \boldsymbol{P}(k) \phi(k)+r_{2}(k)+p_{2}(k) h^{2}(k) \phi^{\mathrm{T}}(k) \boldsymbol{P}(k) \phi(k) \phi^{\mathrm{T}}(k) \boldsymbol{P}(k) \phi(k)=0 .
\end{aligned}
$$

Thus, $r_{2}(k)$ is obtained. Finally, from (4b) and the iteration formula of $\boldsymbol{P}(k)$, requiring $\boldsymbol{M}_{1}(k) \leq \mathbf{0}$ is equivalent to requiring

$$
\begin{aligned}
- & \frac{1}{2}\left[h^{-1}(k-1) \boldsymbol{P}^{-1}(k-1)-h^{-1}(k) \boldsymbol{P}^{-1}(k)\right]+Q_{2}(k)+p_{2}(k-1) \phi(k-1) \phi^{\mathrm{T}}(k-1) \\
= & -\frac{1}{2}\left[h^{-1}(k-1)-h^{-1}(k) \gamma(k-1)\right] \boldsymbol{P}^{-1}(k-1)+Q_{2}(k) \\
& +\left[p_{2}(k-1)+\frac{1}{2} \delta(k-1) h^{-1}(k)\right] \phi(k-1) \phi^{\mathrm{T}}(k-1) \\
\leq & \mathbf{0}
\end{aligned}
$$


from which it is seen that $Q_{2}(k)=0$ makes $\eta^{*}(k)$ the largest solution for $p_{2}(k)$. Note that this choice will meet the p.s.d. constraint on $\Gamma_{2}(k)$ :

$$
\begin{aligned}
\Gamma_{2}(k+1) & =\frac{1}{2} h^{-1}(k) \boldsymbol{P}^{-1}(k)-p_{2}(k) \phi(k) \phi^{\mathrm{T}}(k) \\
& \geq \frac{1}{2} h^{-1}(k+1)\left[\gamma(k) \boldsymbol{P}^{-1}(k)+\delta(k) \phi(k) \phi^{\mathrm{T}}(k)\right] \\
& \geq \mathbf{0} . \quad \square
\end{aligned}
$$

Although these pi-coefficients in (24) may not be the optimal solution for the max- $p$ problem, they can be used to produce good results, as the discussions below show. By setting $h(k)=1 /(2 \gamma(k))$, the pi-coefficients (15) from [5] are

$$
\begin{aligned}
& \left\{\boldsymbol{I}_{2}(k+1), \boldsymbol{Q}_{2}(k), p_{2}(k), r_{2}(k)\right\} \\
& \quad=\left\{\gamma(k) \boldsymbol{P}^{-1}(k)-\delta(k) \phi(k) \phi^{\mathrm{T}}(k),[1-\gamma(k)] \boldsymbol{P}^{-1}(k),-\delta(k), \frac{\phi^{\mathrm{T}}(k) \boldsymbol{P}(k) \phi(k)}{4 \gamma(k)}\right\},
\end{aligned}
$$

and the pi-coefficients in (24) become

$$
\left\{\boldsymbol{I}_{2}(k+1), \boldsymbol{Q}_{2}(k), p_{2}(k), r_{2}(k)\right\}=\left\{\gamma(k) \boldsymbol{P}^{-1}(k)-\eta^{*}(k) \phi(k) \phi^{\mathrm{T}}(k), \mathbf{0}, \eta^{*}(k), \frac{\phi^{\mathrm{T}}(k) \boldsymbol{P}(k) \phi(k)}{4 \gamma(k)}\right\},
$$

where $\eta^{*}(k)$ is the largest $\eta(k)$ satisfying

$$
\gamma(k)[1-\gamma(k+1)] \boldsymbol{P}^{-1}(k)-[\eta(k)+\delta(k) \gamma(k+1)] \phi(k) \phi^{\mathrm{T}}(k) \geq \mathbf{0} .
$$

The setting $\eta(k)=-\delta(k)$ will satisfy $(26)$, hence $\eta^{*}(k) \geq-\delta(k)$, i.e., the pi-coefficients in (24) imply a larger stability range than those in (25). Furthermore, the condition $0<\gamma(k+1)<1$ makes $\eta^{*}(k)>-\delta(k)$. If $\gamma(k)$ is chosen properly, we can even have $\eta^{*}(k)>0$. When $a_{1}=0.85$ and $a_{2}=-0.90$, the FMSG stability condition (22) becomes

$$
r_{0}+\inf _{k} \eta^{*}(k)>0 \text { and } \inf _{k} \frac{\phi^{\mathrm{T}}(k) \boldsymbol{P}(k) \phi(k)}{4 \gamma(k)}>\left|0.75+0.5625 \cdot r_{0}\right|,
$$

which reduce the restriction on $\phi(k)$ for small $r_{0}$.

\subsection{Gradient algorithm}

Compared with the least-squares update law, the gradient algorithm has a simpler form,

$$
\hat{\boldsymbol{\theta}}(k+1)=\hat{\boldsymbol{\theta}}(k)+\varepsilon(k) \phi(k) v(k+1)
$$

whose 'state matrices' in the "state equation" (13) are

$$
\left\{A_{2}(k), b_{2}(k), c_{2}^{\mathrm{T}}(k), d_{2}(k)\right\}=\left\{I,-\varepsilon(k) \phi(k),-\phi^{\mathrm{T}}(k), \varepsilon(k) \phi^{\mathrm{T}}(k) \phi(k)\right\} .
$$

For analyzing its FMSG stability, we have the following theorem.

Theorem 2. A feasible solution to (23), which may not be optimal, is

$$
\left\{\boldsymbol{\Gamma}_{2}(k+1), \boldsymbol{Q}_{2}(k), p_{2}(k), r_{2}(k)\right\}=\left\{\frac{1}{2} \varepsilon^{-1}(k) \boldsymbol{I}-\eta^{*}(k) \phi(k) \phi^{\mathrm{T}}(k), \mathbf{0}, \eta^{*}(k), \frac{1}{2} \varepsilon(k) \phi^{\mathrm{T}}(k) \phi(k)\right\},
$$

where $\eta^{*}(k)$ is the largest $\eta(k)$ satisfying

$$
\frac{1}{2}\left[\varepsilon^{-1}(k)-\varepsilon^{-1}(k+1)\right] I-\eta(k) \phi(k) \phi^{\mathrm{T}}(k) \geq 0 .
$$


Proof. This is a special case of Theorem 1, as can be seen by setting $h(k)=\varepsilon(k)$ and $\boldsymbol{P}(k)=\boldsymbol{I}$, with $\gamma(k) \equiv 1$ and $\delta(k) \equiv 0$.

For the gradient algorithm with fixed step size, i.e., $\varepsilon(k)=$ constant, $\eta^{*}(k)$ must be zero, which implies that its $r_{2}(k)$ and $p_{2}(k)$ cannot be positive at the same time. On the other hand, the least-squares method allows both $r_{2}(k)$ and $p_{2}(k)$ to be positive, provided $\gamma(k)$ and $\delta(k)$ are chosen carefully. In this case, our analysis results reveal that the system with gradient update algorithm preserves smaller stability range than that with least-squares algorithm.

\subsection{The effect of $\sigma$-modification}

In practice, the adaptive systems will encounter some unmodeled dynamics and/or perturbations, which will make the performances of the previous update laws poor. To attain robustness, the update laws have to be modified by some schemes, such as $\sigma$-modification [2]. The gradient algorithm with $\sigma$-modification is

$$
\tilde{\boldsymbol{\theta}}(k+1)=\tilde{\boldsymbol{\theta}}(k)-\varepsilon(k) \phi(k) \boldsymbol{e}(k)-\sigma \tilde{\boldsymbol{\theta}}(k)=(1-\sigma) \tilde{\boldsymbol{\theta}}(k)-\varepsilon(k) \phi(k) \boldsymbol{e}(k), \quad 0<\sigma<1,
$$

whose 'state matrices' in the 'state equation' (13) are

$$
\left\{\boldsymbol{A}_{2}(k), \boldsymbol{b}_{2}(k), c_{2}^{\mathrm{T}}(k), d_{2}(k)\right\}=\left\{(1-\sigma) I,-\varepsilon(k) \phi(k),-(1-\sigma) \phi^{\mathrm{T}}(k), \varepsilon(k) \phi^{\mathrm{T}}(k) \phi(k)\right\} .
$$

For analyzing its FMSG stability, we have the following theorem.

Theorem 3. A feasible solution to (23), which may not be optimal, is

$$
\left\{\boldsymbol{\Gamma}_{2}(k+1), \boldsymbol{Q}_{2}(k), p_{2}(k), r_{2}(k)\right\}=\left\{\frac{1}{2} \varepsilon^{-1}(k) \boldsymbol{I}-\eta^{*}(k) \phi(k) \phi^{\mathrm{T}}(k), \mathbf{0}, \eta^{*}(k), \frac{1}{2} \varepsilon(k) \phi^{\mathrm{T}}(k) \phi(k)\right\},
$$

where $\eta^{*}(k)$ is the largest $\eta(k)$ satisfying

$$
\frac{1}{2}\left[\varepsilon^{-1}(k)-(1-\sigma)^{2} \varepsilon^{-1}(k+1)\right] I-\eta(k) \phi(k) \phi^{\mathbf{T}}(k) \geq \mathbf{0} .
$$

Proof. This proof is similar to those of Theorems 1 and 2.

Comparing (32) with the previous results, we know that the pi-coefficients of the gradient algorithm with $\sigma$-modification can have $\eta^{*}(k)>0$, even with the step size $\varepsilon(k)$ fixed. Hence, the pi-coefficients $r_{2}(k)$ and $p_{2}(k)$ may both be positive. The magnitude of $\eta^{*}(k)$ is directly related to the quantity of the modification $\sigma$.

Finally, consider the least-squares method with $\sigma$-modification. The 'state matrices' of 'state equation' (13) become

$$
\left\{\boldsymbol{A}_{2}(k), \boldsymbol{b}_{2}(k), \boldsymbol{c}_{2}^{\mathrm{T}}(k), d_{2}(k)\right\}=\left\{(1-\sigma) \boldsymbol{I},-h(k) \boldsymbol{P}(k) \phi(k),-(1-\sigma) \phi^{\mathrm{T}}(k), h(k) \boldsymbol{\phi}^{\mathrm{T}}(k) \boldsymbol{P}(k) \phi(k)\right\}
$$

after adding $\sigma$-modification with $\sigma \in(0,1)$. For analyzing its FMSG stability, we have the following theorem.

Theorem 4. A feasible solution to (23), which may not be optimal, is

$$
\begin{aligned}
& \left\{\boldsymbol{I}_{2}(k+1), \boldsymbol{Q}_{2}(k), p_{2}(k), r_{2}(k)\right\} \\
& \quad=\left\{\frac{1}{2} h^{-1}(k) \boldsymbol{P}^{-1}(k)-\eta^{*}(k) \boldsymbol{\phi}(k) \phi^{\mathrm{T}}(k), \mathbf{0}, \eta^{*}(k), \frac{1}{2} h(k) \phi^{\mathrm{T}}(k) \boldsymbol{P}(k) \phi(k)\right\},
\end{aligned}
$$

where $\eta^{*}(k)$ is the largest $\eta(k)$ satisfying

$$
\frac{1}{2}\left[h^{-1}(k)-(1-\sigma)^{2} h^{-1}(k+1) \gamma(k)\right] \boldsymbol{P}^{-1}(k)-\left[\eta(k)+\frac{1}{2}(1-\sigma)^{2} h^{-1}(k+1) \delta(k)\right] \boldsymbol{\phi}(k) \boldsymbol{\phi}^{\mathrm{T}}(k) \geq \mathbf{0} .
$$

Proof. This proof is similar to those of Theorems 1 and 2. 
Compare the two $\eta^{*}(k)$ 's in Theorems 1 and 4. From the inequalities they must satisfy, respectively, we see that the one in Theorem 4 should have a larger positive range, since $(1-\sigma)^{2} \in(0,1)$. That is to say, the least-squares update law with $\sigma$-modification may have more positive $r_{2}(k)$ and $p_{2}(k)$ to compensate for the possible non-SPR of the subsystem $\Xi_{1}$ than that without.

\section{Conclusions}

In this paper, pi-sharing theory is used to study the FMSG stability of a parameter estimation adaptive system, whose error model includes an LTI subsystem and an NTV update rule in a feedback configuration. For the linear subsystem, a set of suboptimal pi-coefficients are found and shown to be more suitable for analysis purposes. For the nonlinear time-varying update laws, a constrained max- $p$ problem is formulated for solving useful pi-coefficients. From the use of these coefficients, the quantitative results show that the system with the least-squares algorithm has a larger stability region than that with the gradient algorithm, and $\sigma$-modification gives both algorithms larger stability ranges.

\section{References}

[1] B.D.O. Anderson et al., Stability of Adaptive Systems - Passivity and Averaging Analysis (MIT Press, Cambridge, MA, 1986).

[2] K.J. Aström and B. Wittenmark, Adaptive Control (Addison-Wesley, New York, 1989).

[3] J.-S. Heh, I.-K. Fong and J.-F. Wu, Optimization of pi-coefficients, Proc. Amer. Control Conf., Chicago, IL (1992).

[4] Y.D. Landau, Adaptive Control - The Model Reference Approach (Marcel Dekker, New York, 1979).

[5] D.A. Lawrence and C.R. Johnson, Jr., Recursive parameter identification algorithm stability analysis via pi-sharing, IEEE Trans Automat. Control 31 (1986) 14.

[6] J.-F. Wu, J.-S. Heh and I.-K. Fong, On computing pi-coefficients, Systems Control Lett. 16 (1991) $457-463$.

[7] J.-F. Wu, J.-S. Heh and I.-K. Fong, The optimal pi-coefficients of second-order systems, J. Chinese Inst. Engrs. 15 (1992) $179-184$. 